\title{
70 Jahre unbemerkt im Keller
}

\section{Wiederentdeckung einer Soldaten-Skulptur der "Weltkriegsbücherei"}

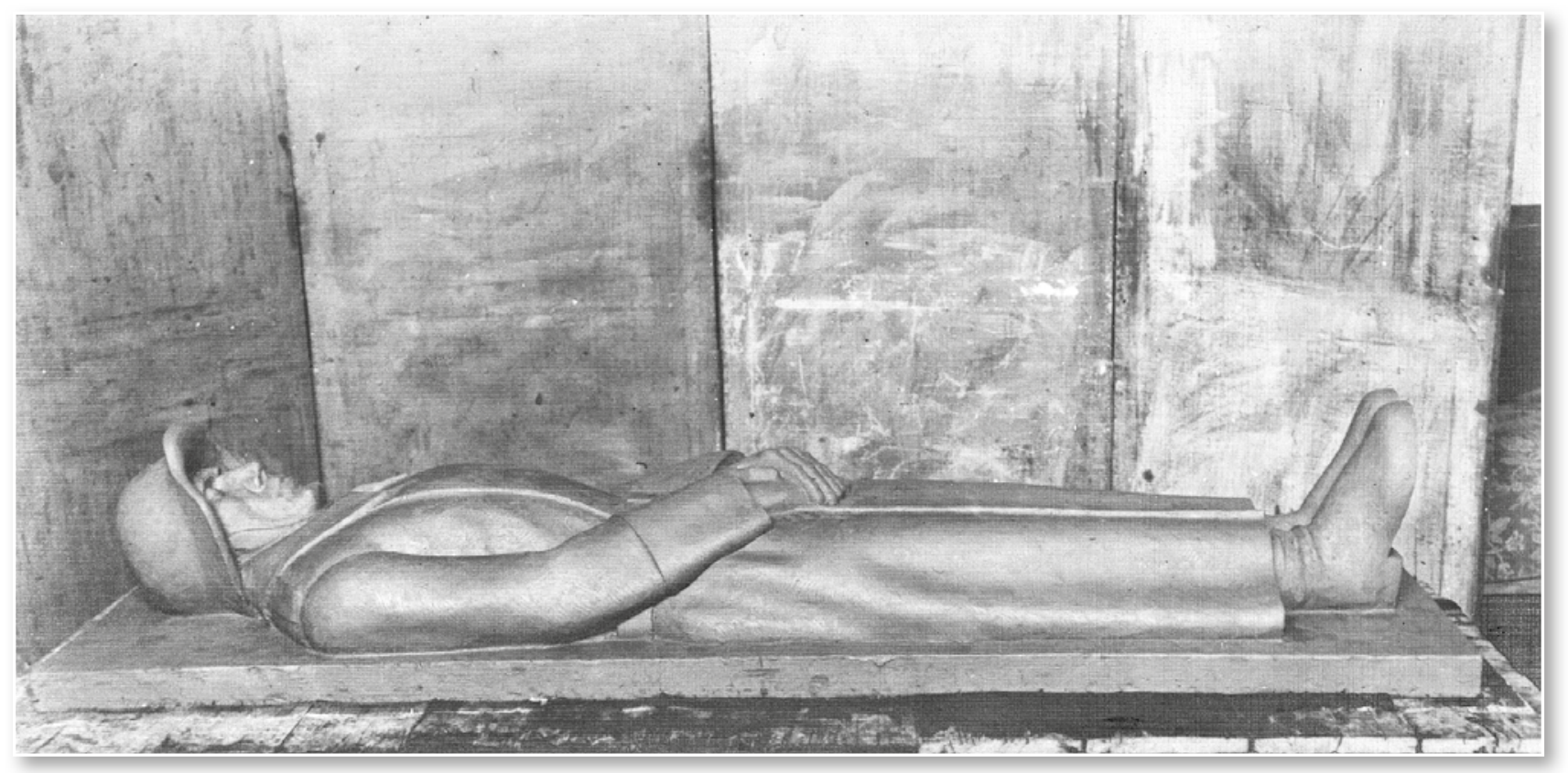

Abb. 1: Soldaten-Skulptur von Peter Hartmann vor der Installation in Schloss Rosenstein

Ausstellungseröffnungen sind eigentlich keine Momente, bei denen man als Kurator größere Überraschungen zum ausgestellten Thema erwartet. Inhaltliche Neuentdeckungen gab es durchaus bei der Vorbereitung der Ausstellung "100 Jahre Bibliothek für Zeitgeschichte. 1915-2015“. Insbesondere zur Zeit des Nationalsozialismus kamen viele bislang unbekannte Informationen ans Licht. Zahlreiche neue Erkenntnisse aus verschiedenen Archiven flossen auch in die Festschrift zum 100-jährigen Jubiläum der Bibliothek ein. Doch dass die Eröffnung selbst ein ganz neues Kapitel der Bibliotheksgeschichte aufschlagen würde, damit hatte niemand gerechnet. Und doch kam es durch eine Verkettung von Zufällen dazu.

Unter den zahlreichen Gästen, die am 19. November 2015 an der Eröffnungsfeier teilnahmen, war auch Markus Speidel, der derzeit am Ausstellungskonzept des Stadtmuseums Stuttgart mitwirkt. Beim Blättern in der Festschrift der Bibliothek für Zeitgeschichte $(\mathrm{BfZ})^{1}$ blieb sein Blick an einem Foto hängen, dessen Motiv ihm seltsam bekannt vorkam. Eine etwa 1,80 m große Soldaten-Skulptur aus Kunststein, „Erinnerungsdenkmal für die gefallenen Deutschen" betitelt, liegt auf einem Sockel in der Eingangshalle von Schloss Rosenstein, aufgenommen in den 1930er Jahren. Am nächsten Morgen fiel Speidel ein, wo er diese Statue schon einmal gesehen hatte: in der Untertürkheimer Zeitung vom 18. Januar 2012. Eine erstaunliche Rekonstruktionsleistung, wenn man bedenkt, dass die Soldaten-Figur auf dem Foto in der Zeitung stehend, aus einem anderen Blickwinkel heraus und in Farbe zu sehen ist!

Anlass für den Zeitungsbericht war, dass das Gefallenendenkmal am 16. Dezember 2011 im Keller des Kindergartens "Kapellenzwerge" in Rotenberg aufgetaucht war. Dort hatte der Soldat der Leiterin des Kindergartens einen ordentlichen Schrecken eingejagt, als er beim Renovieren hinter einem Regal zum Vorschein kam. Sie kontaktierte den örtlichen Bürgerverein, der im oberen Stockwerk des Gebäudes das Ortsmuseum Untertürkheim/ Rotenberg betreibt. Gemeinsam mit der Untertürkheimer Zeitung wurde der Versuch unternommen, Informationen über den Steinsoldaten zu sammeln, 
doch niemand konnte die Skulptur zuordnen. Auch Markus Speidel vom Stadtmuseum nicht, zumindest nicht bis zum 19. November 2015. Am Tag nach der Ausstellungseröffnung machte er die BfZ auf seine Entdeckung aufmerksam - und erregte dort großes Aufsehen. Denn dass das Kriegerdenkmal der Weltkriegsbücherei noch immer existiert, hatte niemand für möglich gehalten, zumal Schloss Rosenstein 1944 von alliierten Bomben getroffen und völlig ausgebrannt war. Außergewöhnlich ist sicher auch, dass ein öffentliches historisches Objekt dieser Größe über Jahrzehnte unbemerkt blieb.

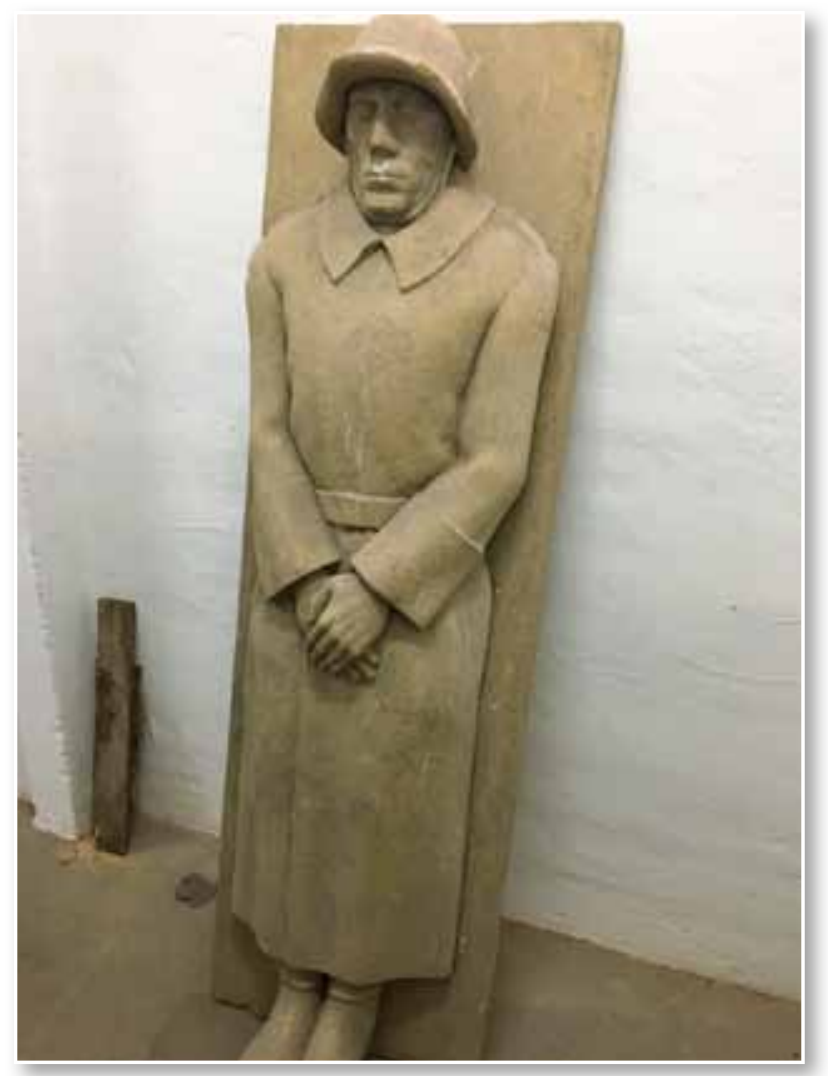

Nachdem "Identität" und Provenienz der Steinfigur geklärt sind, ist nun auch klar, welche neue Heimat die Skulptur finden wird. Da in der Württembergischen Landesbibliothek keine angemessene Unterbringung möglich ist, wird sie demnächst als Dauerleingabe an das Haus der Geschichte BadenWürttemberg gehen. Geplant ist, das Denkmal dort in die überarbeitete Dauerausstellung zu integrieren.

Nach wie vor ist ungeklärt, wie der Soldat vom Schloss Rosenstein in den Keller des Kindergartens gelangte. Die Plastik ist erstaunlich gut erhalten

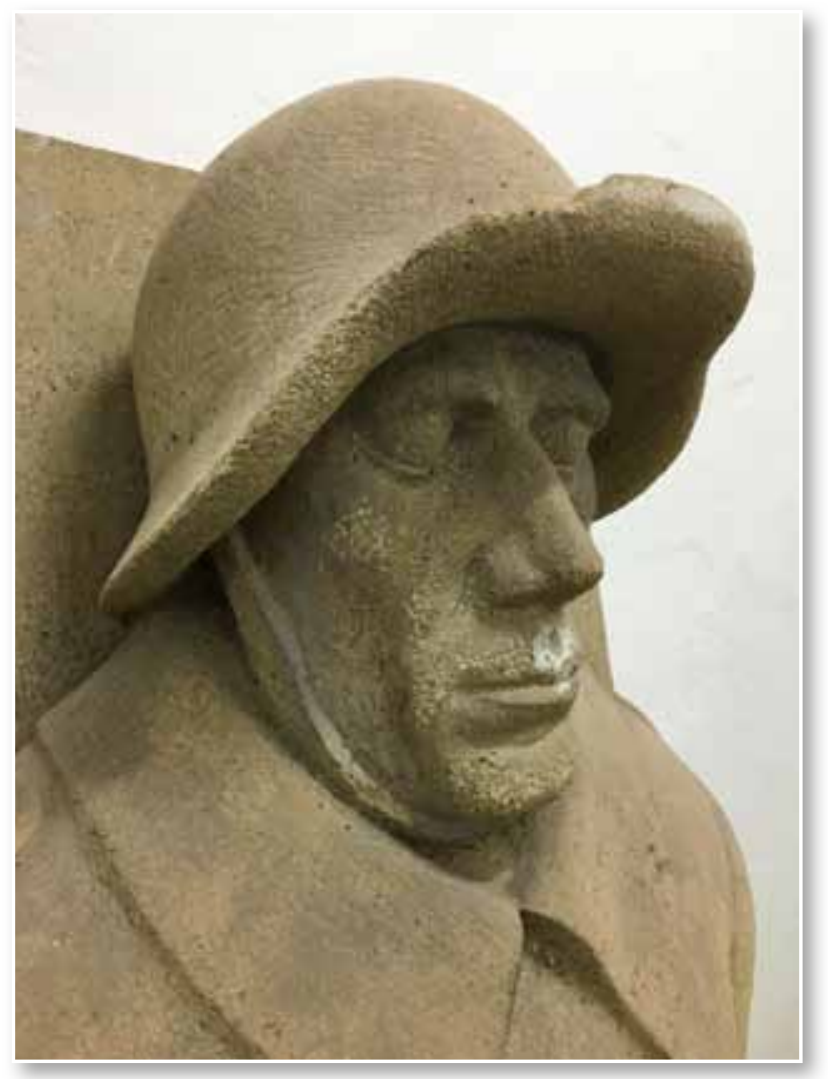

Abb. 2 und 3: Die Soldaten-Skulptur 2015 im Keller des alten Schulhauses in Rotenberg

Inzwischen konnte geklärt werden, dass es sich bei der Skulptur in Rotenberg tatsächlich um das „Kriegerdenkmal“2 handelt, das der Stuttgarter Bildhauer Peter Hartmann Anfang der 1930er Jahre für das Weltkriegsmuseum anfertigte. 1933 war für Friedrich Felger, den ersten Direktor der Weltkriegsbücherei, wie die BfZ bis 1948 hieß, ein Traum in Erfüllung gegangen: Basierend auf ihren umfangreichen Sammlungen eröffnete die Bibliothek im linken Flügel von Schloss Rosenstein ein Museum zum Ersten Weltkrieg. Die Vorhalle des "Kriegsmuseums" dominierte Hartmanns Skulptur, die einen „toten Krieger in Lebensgröße” darstellt. und weist, soweit erkennbar, keine Spuren auf, die auf die Brandkatastrophe vom 12./13. September 1944 hindeuten würden. Aller Wahrscheinlichkeit nach ist sie also bereits vorher nach Rotenberg transportiert worden. Dass das Kriegerdenkmal während des Zweiten Weltkriegs ausgelagert wurde, um es vor den zunehmenden alliierten Luftangriffen zu schützen, erscheint ebenfalls unwahrscheinlich, denn es gelang der Bibliothek nicht einmal, alle Buchbestände rechtzeitig abzutransportieren. Warum hätte man also ausgerechnet eine schwere Skulptur fortschaffen sollen - zumal nach Rotenberg, wo sie wegen der nahen 
Daimler-Werke vor Luftangriffen keineswegs sicher war. Vermutlich wurde die Skulptur bereits in den 1930er Jahren aus Schloss Rosenstein entfernt. Eine mögliche Erklärung könnte sein, dass sie den Nationalsozialisten nicht martialisch genug war der Soldat ist nicht nur unbewaffnet und tot dargestellt, er wirkt auch wenig heroisch. Doch warum gerade Rotenberg? Diese Frage bleibt weiterhin unbeantwortet.

Die umfangreiche Berichterstattung in der Presse ${ }^{3}$ hat aber an anderer Stelle zu neuen Erkenntnissen geführt: Deutlich mehr wissen wir nun über Peter Hartmann, der die Skulptur geschaffen hat. Seine Töchter Sigrune Müller und Reingard Irsigler wurden auf den Fall aufmerksam und lieferten der Bibliothek interessante Informationen über den 50 bisher wenig bekannten Künstler.

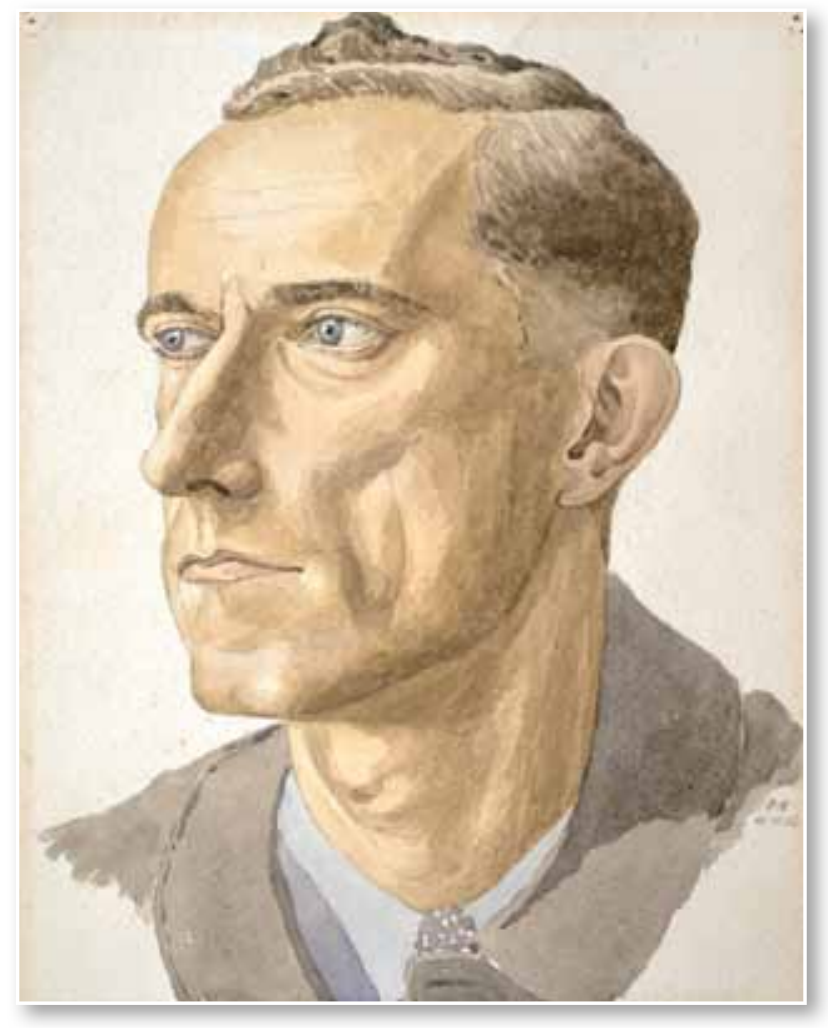

Abb. 4: Peter Hartmann, Selbstbildnis von 1932. Aquarell aus der Skizzenmappe im Nachlass
Peter Hartmann wurde am 21. März 1897 in Weiden in der Oberpfalz (Bayern) geboren. Er nahm als Soldat am Ersten Weltkrieg teil, unter anderem diente er am Hartmannsweiler Kopf in den Vogesen. Nach dem Krieg studierte er Bildhauerei an der Württembergischen Akademie der bildenden Künste in Stuttgart. 1933 war er Mitglied der ständigen Künstlerkommission für Plastik des Württembergischen Kunstvereins und hatte in dieser Funktion die beim Kunstverein eingehenden bildhauerischen Werke zu begutachten. ${ }^{4}$ Möglicherweise ergab sich hier eine Bekanntschaft mit Friedrich Felger, dem ebenfalls künstlerisch ambitionierten ersten Direktor der Weltkriegsbücherei. Eine in Kunststein ausgeführte Skulptur ist eher ungewöhnlich für das Werk Hartmanns, der seinen Töchtern vor allem mit Holzschnitzarbeiten in Erinnerung geblieben ist. Über seine künstlerische Arbeit hat er später wenig gesprochen. Hierzu könnte beigetragen haben, dass er von der Kunst nicht leben konnte und sich daher anderen beruflichen Tätigkeiten widmete. Für den Lebensunterhalt der Familie sorgte vor allem seine Ehefrau Elisabeth Hartmann, die als Sängerin an der Stuttgarter Oper engagiert war. Im Zweiten Weltkrieg wurde Hartmann schließlich erneut eingezogen und kehrte erst 1946 schwer gezeichnet zurück. In französischer Kriegsgefangenschaft hatte er in einem Bleibergwerk in den Pyrenäen arbeiten müssen, was seine Gesundheit stark angriff. Am 29. August 1968 verstarb er mit 71 Jahren in seinem Haus in der Altenbergstraße 42 in Stuttgart. Eine Skizzenmappe, die Einblick in sein künstlerisches Schaffen gibt, befindet sich noch heute im Besitz der Familie. Sie enthält auch zwei Fotos des von inm geschaffenen Kriegerdenkmals.

\section{Christian Westerhoff}

